\title{
Pratiques
}

Linguistique, littérature, didactique

$155-156 \mid 2012$

Lexique et écriture

\section{Favoriser l'accès lexical en situation de production écrite}

Anne Sardier

\section{(2) OpenEdition}

1 Journals

Édition électronique

URL : http://journals.openedition.org/pratiques/3490

DOI : 10.4000/pratiques.3490

ISSN : 2425-2042

Éditeur

Centre de recherche sur les médiations (CREM)

Édition imprimée

Date de publication : 15 décembre 2012

Pagination : 127-146

\section{Référence électronique}

Anne Sardier, « Favoriser l'accès lexical en situation de production écrite », Pratiques [En ligne], 155-156 | 2012, mis en ligne le 18 décembre 2017, consulté le 14 novembre 2019. URL : http:// journals.openedition.org/pratiques/3490; DOI : 10.4000/pratiques.3490

(c) Tous droits réservés 


\title{
Favoriser l'accès lexical en situation de production écrite
}

\author{
Anne Sardier \\ IUFM d'Auvergne, Université Blaise Pascal, \\ antenne d'Aurillac. \\ LIDILEM, E.A. 609, Université de Grenoble
}

Les relations entre lexique et production d'écrit subordonnent souvent l'un à l'autre ces deux aspects de l'enseignement du français. Le lexique peut être considéré seulement comme un instrument à destination de la production d'écrit. Dans ce cas le lexique est abordé dans une perspective utilitariste et il est censé constituer un des éléments permettant d'améliorer la qualité de la production par un recours à des unités lexicales plus riches et plus justes. Se pose ainsi la question du " mot juste » comme le remarque M.-A. Paveau (2000 : 22), notamment lorsque l'on se rend compte qu'il n'existe pas forcément un mot juste, mais souvent plusieurs, en fonction des choix effectués par le scripteur (Grossmann et Boch, 2003). Cette question du choix opéré par le scripteur est centrale dans l'enseignement-apprentissage du lexique. Le travail de l'enseignant consistera davantage à enseigner à effectuer ces choix qu'à trouver un seul mot juste susceptible de convenir.

Néanmoins, les différents programmes concernant l'enseignement du français, tant à l'école primaire qu'au collège, tendent depuis plusieurs années à proposer plutôt une autre approche du lexique en en faisant un objet d'enseignement-apprentissage à part entière. Il est possible de retrouver dans les manuels les conséquences de ces nouvelles orientations et les propositions faites aux enseignants présentent alors des séances bien spécifiques consacrées au lexique. Mais là encore des difficultés subsistent. Comme le font remarquer F. Grossmann et F. Boch (2003), le travail effectué sur le lexique, sur la grammaire également, est souvent déconnecté de toute activité de production. Dans ce cas, la question se pose de l'intérêt des activités présentées, si l'élève ne met pas à l'épreuve ses nouveaux acquis. Quelle finalité peut-il accorder à des activités sur le lexique si on ne lui fournit pas l'occasion d'employer en situation de production ses nouvelles découvertes? Les manuels présentent aussi très majoritairement des séances grandement axées sur la morphologie, parfois sur le sens, mais fort peu permettent d'effectuer un lien entre ces deux éléments structurant le lexique. Là se pose donc la question de la structuration du lexique, de l'organisation de son enseignement et ainsi de la place de la production, éminemment liée au sens.

Par ailleurs, nous avons pu remarquer dans un travail antérieur (Sardier \& 
Grossmann, 2010) sur le réemploi lexical, que différents éléments interviennent tous de manière déterminante dans l'acquisition du lexique, notamment la production d'écrit. Il semble alors essentiel, considérant les avancées en matière de didactique du lexique ces dernières années, de mieux situer la place de la production dans cet enseignement-apprentissage. Ainsi, au lieu de percevoir la production comme prétexte à étudier des éléments du lexique, nous nous sommes demandé dans quelle mesure les situations de production ne seraient pas des éléments concourant à l'acquisition du lexique. Les élèves passant progressivement de la compréhension à la production (David, 2000), de l'oral à l'écrit, en prévoyant « des démarches favorisant une interaction entre pensée, langage et action » (E. Calaque, $2000: 24$ ), la question soulevée peut être ici celle d'une nécessaire interaction entre production orale et production écrite dans l'appropriation du lexique. Il s'agit alors de savoir comment faire pour que la production orale et les interactions verbales constituent un facteur déterminant de l'accès lexical en situation de production écrite.

Afin de mieux définir le statut des situations de production dans un travail lexical, nous reviendrons dans un premier temps sur les éléments structurant l'enseignement-apprentissage du lexique. Puis dans un deuxième temps, nous proposerons et analyserons des mises en œuvre d'enseignement du lexique prenant en compte production orale et écrite, afin de voir dans quelle mesure ces situations pourraient en effet déclencher l'accès lexical.

\section{Les éléments structurant l'appropriation lexicale}

Comme nous l'avons déjà remarqué (Sardier \& Grossmann, 2010), il existe deux paradigmes explicatifs de l'appropriation du lexique qui sont liés soit à ses structures, soit à son emploi discursif en situation de communication. En effet, il s'agit à la fois de comprendre et mettre en œuvre les structures régissant le lexique, et, aspect déterminant, d'effectuer un emploi pertinent en contexte. Ces aspects sont liés à la sémantique et la morphologie, aux relations syntagmatiques qu'entretiennent entre elles les unités lexicales, au contexte d'emploi.

\subsection{Le problème de la référence}

Guy Legrand (2004 : 25), dans son analyse les recommandations officielles, remarque que les propositions de structuration lexicales « portent de manière très privilégiée sur quatre relations sémantiques et formelles de base (synonymie, antonymie, dérivations, thème ou champ lexical), sur une exploration du sens des mots par définition, parcours du champ sémantique, et, plus récemment, distinction du sens propre et du sens figuré, en relation avec les contextes, [...]. Des activités de classement confortent ces études ». Nous retrouverons plus loin dans le Bulletin Officiel de juin 2008 ces axes en ce qui concerne les compétences liées au lexique : «Acquisition du vocabulaire / Maîtrise du sens des mots / Les familles de mots / Utilisation du dictionnaire », avec un accent mis sur l'aspect morphologique. Il nous semble important d'orienter la didactique du lexique dans d'autres directions que les quatre relations traditionnellement explorées, en prenant mieux en compte l'hyperonymie, la méronymie, et en reliant davantage l'étude morphologique encore sous-utilisée à la réflexion sur le sens, en langue et en discours, de manière à optimiser le réemploi du lexique nouvellement acquis en situation de communication. Dans une perspective de réemploi, il faut que l'élève construise et structure son lexique interne, mais il est également nécessaire qu'il le réinvestisse en situation de production parce que ce n'est qu'à ce moment-là que le réemploi sera effectif. 
Par ailleurs, se pose ici le problème crucial de la conceptualisation et de l'accès à la référence. G. Petit (2000 : 55) définit la notion d'Unité Lexicale comme « toute séquence qui [...] entretient une relation stable et récurrente avec un élément du réel, qui nécessite un apprentissage et une mémorisation, c'est-à-dire qui ne présente pas une signification accessible uniquement par décomposition du signifiant ». Les élèves ont parfois des difficultés à se représenter ce contenu sémantique des unités lexicales car ils ne possèdent pas de représentation suffisante du concept auquel il est fait référence. Il s'agit donc d'aider les élèves à mettre en rapport les significations du mot avec leur expérience propre. Ainsi, la remarque de G. Petit nous amène à nous questionner sur ce problème de conceptualisation. Les élèves peuvent ainsi accéder au sens courant de certaines unités lexicales, sans les comprendre en contexte car elles peuvent alors faire référence à des concepts qu'ils ne maitrisent pas. Nous le verrons dans les séances que nous avons mises en œuvre, notamment pour l'adjectif construit « infortuné » employé par Homère dans l'Odyssée et associé à Ulysse. Il s'agira donc de proposer des situations permettant aussi aux élèves de s'approprier ces concepts. Le problème de cette nécessaire conceptualisation nous amène à envisager la construction de la référence dans le discours, car c'est là que l'unité lexicale prend tout son sens ; cette difficulté de conceptualisation est d'ailleurs relevée par E. Calaque (2000) ${ }^{(1)}$.

Dans ce cadre, l'influence du co-texte et du contexte dans le «calcul du sens » (Guimier, 1996) a été maintes fois rappelée par les linguistes : elle est liée à l'emploi en situation de discours donc en situation de compréhension et de production. Dominique Maingueneau rappelle d'ailleurs que « le contexte joue un rôle essentiel dans la production comme dans l'interprétation des énoncés » (Maingueneau, 1996 puis 2009 : 33). Au co-texte viendront en effet s'ajouter entre autres la valeur sémantico-référentielle des unités lexicales. L'importance allouée, dans l'enseignement, au contexte d'emploi et au co-texte va pouvoir déterminer le statut de la situation de production.

Nous envisageons donc des situations d'enseignement-apprentissage du lexique où l'ensemble de ces éléments puisse être pris en compte. Dans cette perspective, la production pourrait permettre l'accès lexical parce qu'elle est aussi le moment où l'élève établit, essaie des combinaisons entre unités lexicales pour exprimer sa pensée. Tout en considérant les aspects morphologiques et sémantiques, il s'agit ainsi d'aborder l'enseignement-apprentissage du lexique également sous cet angle syntagmatique.

\subsection{La dimension syntagmatique}

Les relations que les éléments lexicaux d'un énoncé entretiennent entre eux méritent sans doute d'être mieux prises en compte dans l'enseignement-apprentissage du lexique. Depuis les travaux de Tesnière (1959), Fillmore (1968), Picoche (1986, 1993), Mel'čuk (2004), les didacticiens du lexique ont commencé à prendre en compte l'importance des actants dans l'acquisition du vocabulaire. Une piste didacti-

(1) Nous voulons cependant dire que la construction d'un concept n'implique pas de refuser toute "décomposition du signifiant », c'est-t-à dire toute analyse morphologique. Sans doute est-il possible, sans pour autant évincer une nécessaire approche morphologique, de passer de séances où l'on construit la référence en discours à des séances où, pour structurer l'apport lexical, on analyse les lexèmes et où on peut aussi prendre en compte les combinaisons apparaissant sur l'axe syntagmatique. Cette structuration basée sur les combinaisons pourrait alors consolider les nouvelles acquisitions et permettre ainsi le réemploi en situation de production. 
que consiste à travailler avec les élèves sur les structures actancielles de certains mots en contexte afin qu'ils en saisissent mieux les sens et donc puissent mieux se les approprier. Expliciter le rôle des actants présents dans l'énoncé peut permettre d'amorcer une désambiguïsation du sens. Les élèves s'approprient effectivement les différents sens d'un mot et ils ont alors la possibilité de les employer en situation de production.

Plus récemment Polguère $(2004,2008)$ a rappelé le rôle essentiel des actants dans la modélisation des fonctions lexicales en remarquant que ces fonctions étaient notamment basées sur les notions d'actants et de prédicat sémantique. Il est donc difficile de considérer le mot isolément, d'estimer qu'il n'est pas besoin de proposer aux élèves des situations de production pour qu'ils parviennent à accéder au lexique disponible, voire en cours d'appropriation.

Par ailleurs, le recours aux combinaisons entre les unités lexicales apparait essentiellement en situation de discours, donc en situation de production. Il existe une interdépendance, une interaction constante entre acquisition du lexique, compréhension et production. Compte tenu de la prise en compte des différentes structures régissant le lexique, mais aussi de la dimension sémantique et discursive de l'appropriation lexicale, il est impossible de faire abstraction de toute situation de production dans l'enseignement-apprentissage du lexique. L'étude du lexique se trouve ainsi liée à la production et la didactique du lexique n'est plus prisonnière « d'une entrée lexicale centrée sur le mot » (Chanfrault-Duchet, 2004 : 103) à chaque séance de classe. Néanmoins, ce travail sur l'axe syntagmatique et sur les combinaisons possibles pose aussi la question des normes, à la fois culturelles et linguistiques, à partir desquelles il sera envisageable ou non de produire différentes associations. Ce travail axé sur les combinaisons de certains mots entre eux va en effet poser le problème de ce qui est possible et de ce qui ne l'est pas. Ainsi par exemple, il ne sera pas possible d'associer à n'importe quelle épithète homérique n'importe quel personnage ; là interfèrent très directement le travail d'association mené sur l'axe syntagmatique et le travail relatif au contexte culturel. Compréhension et lexique interagissent l'un sur l'autre. En effet, pour ce qui concerne les épithètes homériques que nous avons étudiées en classe de $6^{\mathrm{e}}$, le récit, les relations entre les personnages, etc., déterminent l'emploi de telle ou telle épithète.

\section{Une situation d'enseignement-apprentissage en classe de $6^{\mathrm{e}}$}

Comme nous l'avons précisé plus haut, nous souhaitons mettre l'accent dans les mises en œuvre sur cette interaction entre les situations de production orales et écrites dans l'enseignement - apprentissage du lexique. Nous tenons compte du fait que le réemploi du lexique s'effectue d'abord à l'oral puis à l'écrit (David, 2000). Il nous faut donc prévoir des séances permettant aux élèves de s'approprier progressivement de nouvelles unités lexicales, leur permettant également de les réemployer d'abord en situation orale, puis en situation écrite. Nous souhaitons montrer que le fait de se référer à une situation pédagogique mettant en œuvre la production orale et les interactions verbales va aider les élèves à mobiliser le lexique nouvellement acquis pour le réemployer dans des situations écrites.

\subsection{Le dispositif mis en œuvre}

Nous avons travaillé avec Florence Pons, professeure de français qui intervient dans deux classes de $6^{\mathrm{e}}$ d'un collège d'Aurillac. Les deux classes présentent des ni- 
veaux assez semblables. Nous avons mis en œuvre le même nombre de séances dans chacune des classes, au même moment de l'année, mais dans l'une d'elle ont eu lieu des exercices faisant intervenir l'oral, tandis que dans l'autre ont été donnés des exercices écrits.

En accord avec l'enseignante, nous avons sélectionné un corpus d'unités lexicales liées au texte littéraire que les élèves devaient travailler à cette période. Notre choix s'est donc porté sur l'acquisition d'unités présentes dans L'Odyssée d'Homère, texte au programme de la classe de $6^{\mathrm{e}}$. Le travail concerne essentiellement le premier chapitre, "Calypso ». Les élèves des deux classes disposent de l'ouvrage dans la collection des Classiques abrégés de l'École des Loisirs. Le corpus sur lequel nous travaillons est constitué des épithètes homériques présentes dans ce premier chapitre. Ces tournures employées par Homère sont construites par association entre un nom et son complément. Cette association permet de caractériser le personnage concerné en mettant l'accent sur l'une de ses particularités, comme nous le verrons plus loin ${ }^{(2)}$. Les épithètes jouent ainsi un rôle important dans la distinction des personnages qui sont nombreux ; elles déterminent par là l'accès au sens du texte. Néanmoins, ce travail sur les épithètes homériques trouve sa limite compte tenu de la part de figement inhérente à leur construction, puisque on travaille une caractérisation stéréotypique. Par l'emploi de ces tournures conventionnelles, c'est aussi la question de la construction de la référence dans le contexte de l'œuvre qui se pose ici ; l'association effectuée doit pouvoir produire un sens en cohérence avec l'histoire racontée. Le travail d'association, en mettant l'accent sur les rôles et les particularités des personnages, peut permettre de mieux comprendre leurs relations.

Les séances consacrées à l'appropriation de ces unités lexicales respectent une progression précise prenant en compte l'ensemble des paramètres intervenant dans le processus de l'appropriation lexicale (Sardier \& Grossmann, 2010). Nous avons scrupuleusement respecté la même démarche, le même nombre de séances, le même nombre d'exercices (tous identiques à part la distinction sur l'un d'entre eux oral / écrit) dans chacune des deux classes.

Ainsi une première lecture a permis une rencontre des élèves avec les unités lexicales sur lesquelles ils sont amenés à travailler. Afin de faire émerger les éventuelles unités lexicales que les élèves auraient pu acquérir lors de leur lecture personnelle, un travail de reformulation du texte leur a été proposé. Des parties différentes du premier chapitre sont distribuées aux élèves qui travaillent en groupe. Dans chaque groupe, après négociation, les élèves proposent une reformulation écrite. Chaque production est ensuite lue à la classe afin que le texte soit remis dans l'ordre par négociation de tous. Cette première séance peut ainsi permettre aux élèves d'approfondir la compréhension du texte ; elle permet aussi de faire émerger le lexique disponible au moment de la reformulation, avant d'aller vers l'acquisition d'autres unités lexicales, ce qui est une des conditions permettant l'appropriation du lexique. Cette séance permet à l'enseignante de demander une première production écrite. L'interdépendance entre compréhension, production et lexique disponible est ainsi mise en évidence dans cette première phase du dispositif.

Dans la deuxième phase du dispositif, un travail explicite sur le corpus lexical lié aux épithètes homériques a lieu. Dans un premier temps un relevé des personnages importants est effectué et les groupes nominaux correspondants sont consignés au tableau par l'enseignante. Il s'agit alors de procéder à un relevé d'occurrences concer-

(2) Ainsi par exemple «Zeus, l'assembleur des nuées », « la divine Calypso », « Poséidon, le puissant qui ébranle la terre »... 
nant les désignations des personnages ; les élèves disposent ainsi d'un matériau linguistique mis au jour pour pouvoir le manipuler par la suite. Le travail se fait explicitement sur ce corpus associant des groupes épithètes aux noms des personnages. Dans un deuxième temps, les exercices portent sur l'exactitude référentielle dans le contexte de l'œuvre, ainsi que sur la relation syntagmatique. Ces exercices d'association permettent en effet de mettre l'accent sur les différentes constructions possibles des syntagmes nominaux désignant les personnages ; nous en proposons quelquesuns en annexe (annexe 1).

Dans la troisième phase, une différenciation s'opère entre les deux classes concernées. A la suite de ce travail explicite sur le corpus sélectionné, des exercices d'emploi sont proposés aux élèves. Dans une classe le travail s'effectue par écrit et les élèves doivent produire d'autres types d'associations en utilisant des ériques qu'ils doivent employer dans une courte production de phrase. Dans l'autre classe, le travail s'effectue oralement; les élèves doivent proposer à la classe une épithète pour l'un de leurs camarades. Lors de ces productions, ils peuvent donc expérimenter de nouvelles associations, s'essayer à l'emploi de ces nouvelles tournures, pour les uns à l'écrit et pour les autres à l'oral.

Dans la quatrième phase intervient une nouvelle situation de production écrite. Les élèves ont eu à s'exercer dans une production dont la consigne demandait explicitement un réemploi des épithètes. Les élèves ont alors l'occasion de s'essayer à employer les nouvelles unités lexicales en situation de discours écrit. Nous reproduisons en annexe 2 la consigne donnée par l'enseignante. Un retour sur la production permet alors de voir si l'emploi des épithètes choisies par les élèves est cohérent sur le plan référentiel (rôles et caractéristiques des personnages) et sur le plan de la construction syntaxique.

Enfin, une troisième situation de production a été proposée aux élèves. Ils étaient susceptibles d'y employer les nouvelles unités lexicales travaillées, mais cet emploi ne leur était pas demandé explicitement. Cette production est importante afin d'évaluer une réelle appropriation du lexique travaillé. La consigne et la situation de production doivent permettre de déclencher l'accès au lexique. Le dispositif présente donc bien une interaction profonde entre l'appropriation du lexique et la production d'écrit.

Lors de ces mises en œuvre, les données recueillies concernent les échanges en classe, qui ont été filmés, ainsi que les productions écrites des élèves. Pour la production 0 favorisant l'émergence du lexique disponible, les élèves ayant travaillé en groupe, nous disposons de six productions par classe. En ce qui concerne la production 1, les élèves ont travaillé individuellement et dans ce cas nous disposons de 22 copies dans la classe A (classe qui n'a pas pratiqué d'exercice oral) et 19 copies dans la classe B (classe qui a pratiqué l'exercice oral). Enfin pour la production 2 nous disposons également d'une production par élève, soit, ce jour-là, 21 dans la classe A et 20 dans la classe B. Nous aurions pu comparer le nombre d'occurrences des unités lexicales abordées présentes entre les productions 0 et les productions 3 , afin d'évaluer l'écart éventuel ; la mise en œuvre étant cependant très différente dans les deux cas, il nous parait difficile d'établir une analyse comparative de ces deux productions. En revanche, les productions 2 et 3, qui ont suivi un déroulement semblable, peuvent être rapprochées, comme nous le verrons. Nous tenons compte des ces aspects relatifs au déroulement des séances dans nos analyses.

Ainsi dans les deux classes, les mêmes phases ont été mises en œuvre :

- phase 1 : premier contact des élèves avec les unités lexicales, émergence du lexique disponible (production 0 ); 
- phase 2 : travail systématique ('séances de vocabulaire') concernant ces unités lexicales, en prenant en compte les aspects contextuels, sémantiques, syntagmatiques ;

- phase 3 : différenciation par travail d'association en production écrite ou orale ;

- phase 4 : situation de réemploi explicitement demandé en production écrite avec retour sur les textes des élèves (production 1);

- phase 5 : situation de production écrite avec un réemploi non explicitement demandé par l'enseignante (production 2).

Il est important, dans la mesure du possible, que cette chronologie soit respectée et que les différentes phases se succèdent afin de favoriser l'apprentissage des élèves.

Nous pouvons relever cependant que dans la démarche présentée ici, l'aspect morphologique n'apparaît pas. Il devrait être abordé au cours de la phase 2 , mais l'enseignante a choisi de travailler ce point en cours de grammaire dans une séance intitulée « du nom au groupe nominal ». Ce choix est important car il met l'accent sur l'interdépendance entre le travail lexical et le travail grammatical; il est rare que l'un puisse aller sans l'autre. Cependant, lors des interactions verbales au sein des classes, le recours à la morphologie et à l'étymologie a été assez fréquent, comme nous pourrons le voir par la suite.

\subsection{L'analyse des mises en ouvre proposées}

L'analyse comparative des copies et des échanges produits dans les deux classes permet d'établir différents constats quant à l'enseignement-apprentissage du lexique et son lien avec les interactions verbales et la production d'écrit.

\subsubsection{La production écrite comme élément déterminant de l'appropriation lexicale}

L'analyse des interactions verbales et des productions 0 (reformulations écrites) liées à la première phase du travail confirme le lien étroit entre le lexique et la compréhension, souvent rappelé (par exemple David, J., Dubois, D., Fayol, M., Rémond, M. (coord.). (2000) parmi d'autres propositions sur ce sujet). Ainsi les propositions des élèves et leurs justifications révèlent des difficultés de compréhension, mais il semble que c'est bien ce travail interactif entre compréhension et lexique qui a permis de mettre au jour ces problèmes, comme nous le montre l'extrait 1 ci-dessous. Au moment où les élèves reformulent et où un rapporteur présente le résumé de son groupe, une confusion apparait entre la déesse Athéna et Ino aux beaux talons, les élèves ont alors des difficultés à remettre le texte dans l'ordre.

Extrait 1:

Pour le dernier groupe se pose un problème relatif aux personnages et à leur dénomination.

$P \quad$ Vous dites Athéna...

E (dernier groupe) ... Athéna donna le voile à Ulysse

$P$ Athéna donna le voile à Ulysse. Quelqu'un n'en parle pas de ce voile à un moment?

E (groupe 2) Ben c'est pas les mêmes...

$P \quad C^{\prime} e s t$ pas les mêmes, pourquoi c'est pas les mêmes?

$E$ (groupe 2) Ben pour nous c'est Ino aux beaux talons qui donne le voile.

$P \quad$ Ino aux beaux talons qui lui donne... Vous êtes sûrs?

E (dernier groupe) Je peux lire dans le texte? 
P Oui

E «La déesse ayant ainsi parlé lui donna le voile et retourna..."

P Oui, mais la déesse... Pourquoi ça peut être difficile quand on lit "la déesse»?

E Parce que des fois ça peut être Athéna, des fois pas...

$P \quad$ Si on dit juste "la déesse » dans ce chapitre, qui ça peut désigner?

E Athéna ou bien Calypso

P Calypso aussi..., et aussi...

E Ino

$P \quad$ Ino... Voilà, le groupe "la déesse " peut désigner trois personnages. Alors il faut lire très attentivement pour savoir qui ça désigne.

Ce court extrait montre bien le rôle de chaque élément du dispositif. La reformulation écrite et sa restitution orale, le rapport au texte, la réflexion sur le lexique désignant les personnages, vont révéler des difficultés de compréhension. L'élève qui a eu des difficultés concernant l'identité de "la déesse » éprouve le besoin de revenir au texte, mais cela ne suffit pas à résoudre son problème. De cette façon, en posant le problème de l'établissement de la référence pour ce qui est du groupe « la déesse », le dispositif permet de mettre l'accent sur les rôles des personnages et sur leur caractérisation. C'est par l'interaction avec le groupe classe que la difficulté sera levée. Nous analyserons plus loin le rôle déterminant de ces interactions verbales dans ces classes. Cependant, les difficultés des élèves rappellent ici que le travail sur le lexique est éminemment lié au contexte d'emploi, en production et en compréhension. C'est la pluralité de référents auxquels renvoie le groupe « la déesse » qui pose problème et cette difficulté, soulevée lors des propositions de reformulation, ne peut être surmontée qu'avec le recours au contexte de l'histoire. Ainsi, ce n'est pas le sens du groupe «la déesse » qui pose problème aux élèves, mais bien l'établissement de la référence dans le contexte de l'œuvre.

Par ailleurs, les liens entre la production, le lexique et la compréhension apparaissent également au moment où les élèves doivent effectuer leur deuxième production écrite dans laquelle le réemploi est explicitement demandé par l'enseignante. Cette demande explicite entraine bien entendu un recours très systématique aux épithètes homériques, ce qui, d'un point de vue stylistique peut être contestable, mais ce qui permet néanmoins aux élèves de s'essayer à l'emploi des épithètes. Or certaines copies révèlent là des emplois problématiques, comme nous allons le voir. Nous y soulignons les emplois des différentes épithètes. Ainsi la production 1 de Dylan A :

« Une fois qu'Athéna fils de Zeus et Ulysse le dieu des dieux arrivèrent a Ithaque sur l'île des phéaciens aphrodite fils d'Ulysse réussie a vouloir que Ulysse tombe amoureu de Nausicaa la future epouse d'Ulysse qui n'est pas insensible a son charme, e qui vouler a tout prix qu'il tombe amoureu de elle car ils etaient très amoureu l'un que l'autre. »

ou bien la production 1 d'Antoine :

«Athéna protéga Ulysse le tout puissant qui ébranle la terre voulait rentrait chez lui a Ithaque mais Ulysse le tout puissant qui ébranle la terre s'était engagé d'un profonde somel. Lors du séjour d'Ulysse le tout puissant qui ébranle la terre sur l'île des Phéaciens mangeurs d'homme.

Un jour les Phéaciens tueur d'homme se trouvèrent nez à nez avec Ulysse le généreux, Ulysse et Athéna la déesse de la sagesse. Quand tout a coup les Phéaciens tueur d'homme leur coururent après, Ulysse le génér est Athéna fille de Zeus coururent a leur toure...

Ils leur échapperre mais restaire sur leur garde. 
Aphrodite l'arogant intervient et voulut tombé amoureux de Nausicaa la plus belle qui ne résista pas au charme d'Aphrodite le généreux qui fut ensemble pas longtemps après. »

Dans ces copies, certains emplois des épithètes homériques révèlent les difficultés des élèves à se représenter l'identité des personnages et leurs rôles les uns par rapport aux autres. Les confusions d'ordre référentiel, apparaissent notamment dans des désignations telles "Athéna fils de Zeus », "Aphrodite fils d'Ulysse », censées rappeler la filiation des personnages, comme dans l'œuvre d'Homère. De même, les associations du type "Aphrodite l'arogant», ou "Aphrodite le généreux », épithètes qui se contredisent, posent des problèmes d'exactitude référentielle dans le contexte de l'œuvre. La confusion de genre peut, elle aussi, être attribuée à un problème d'identification référentielle. A ce stade, comme nous l'avons dit, les élèves font encore des essais, parfois malencontreux, mais également révélateurs de leurs difficultés. La consigne, en insistant sur l'emploi des épithètes, permet alors de mettre au jour certaines difficultés des élèves dans la compréhension du texte. En revanche, les constructions des associations restent fidèles aux constructions du texte, telles «Ulysse le généreux », ou «Zeus l'assembleur des nuées » utilisées par Homère dans ce premier chapitre.

Certaines copies présentent également des emplois où la référence et la construction syntagmatique ne posent pas de problème. Ainsi la production 1 de Chloé :

"Alors qu'Ulysse le rusé s'échouait sur une plage Phéacienne, il vit devant lui Nausicaa, fille de Alkinoos. Elle l'aida à se relever, puis elle le lava et lui donna des vêtements. Ensuite elle l'emmena chez son père. Pendant qu'ils marchaient Aphrodite, la déesse au grand amour, pénétra dans l'esprit d'Ulysse le généreux et le fit tomber amoureux de Nausicaa. Devant la château d'Alkinoos, Ulysse l'hypnotisé demanda Nausicaa en mariage.

Choquée, Nausicaa lui demanda d'attendre un jour avant qu'elle se décide. Alors qu'elle courait se réfugier dans sa chambre Ulysse alla voir Alkinoos l'hospitalié, lui conta ses aventures, puis, après le diner, lui demanda sa fille en mariage. Alkinoos lui répondit qu'il n'y avait aucun inconvénient.

Pendant ce temps, dans sa chambre, Nausicaa priait pour que tout se passe bien. Le jour suivant, elle dit à Ulysse qu'elle acceptait.

Le roi Alkinoos organisa le mariage, mais, juste avant que ça commence, Athéna la déesse guerrière chassa Aphrodite, qui perdit tout contrôle sur Ulysse.

C'est a ce moment-là qu'il comprit ce qu'il se passait. Il annula le mariage et rentra chez lui à bord d'un bateau de Alkinoos. »

Dans cette production, les épithètes reprennent parfois la lettre du texte, comme « Ulysse le rusé », d'autres sont inventées par l'élève et paraissent adaptées à la production et au contexte de l'œuvre, ainsi par exemple "Alkinoos l'hospitalié » ou « Athéna la déesse guerrière ».

Dans ces premières copies, les essais sont nombreux car demandés dans la consigne. Ainsi, dans un premier temps, il est possible de considérer que la production devient une sorte de prétexte à l'emploi.

En revanche, les deuxièmes productions, dans laquelle l'enseignante ne demandait pas explicitement l'emploi d'épithètes, présentent des essais beaucoup moins nombreux. Ainsi la production de Dylan :

«Arrivé sur l'île des Anticlope Ulysse et ses compagnons eu une peur afrose. Cet île faisait très peur, c'était sombre. Tout à coup Ulysse et ses compagnont se faissait piégé par les Anticlop les habitants de village il était monstrueux il avait de 
tête de tigre 4 mains et 4 pieds d'éléphant et un grand cord de guepard il était horible il avait prit ulysse et ses compagnont prisonier dans le village il était ligoter par de grosses cordes

Ulysse le hero au mille russe attrape un ciseaux par terre et coupa la corde et se libera il tua le maitre de Anticlope en lui enfoncant le ciseaux dans le ventre et il reparti a Ithaque retrouver sa cher et tendre Penelope. »

ou la production $2 \mathrm{~d}$ 'Antoine :

«La ruse d'Ulysse sur l'ile de forestudon.

Ils étaient sur la mer puis ils arrivèrent sur l'île de forestudon.

Ils visitèrent l'île de forestudon quand tout-à-coup les habitants qui étaient des monstres se trouvèrent nez à nez avec Ulysse est sa troupent.

Ils resemblèent à des tigres a 3 têtes et 6 pates.

Les monstres demandèrent le combat, Ulysse est sa troupent acseptèrent le combat. Ulysse l'aisa sa troupent et parti an haut de la falaise il avait avec lui une énorme pière qu'il poussa et la pierre tomba sur un monstre. Les monstes étaient plus que 2 .

Ulysse avait reussi a eliminé 1 monstre. Ulysse repartie avec les siens pour continuer le combat il alla voir si les siens était mort mais ils n'étaient pas mort, Ulysse repartie cette fois dans la forêt mie piège et attirèrent les monstres dedant puis les monstres se sont fient avoir est ils mourrurent 4 jours après plus aucun monstre était en vie.

Ils rentrèrent chez eux et content de ne pas être mort. »

ou celle de Chloé :

«Ulysse, avec ses compagnons, arriva dans une mer hérissée de récifs, crées par Poséidon, sur lesquels étaient plantés des têtes de mort. Ils continuèrent leur avancée, terriffiés. Ils accostèrent sur un île peuplée de monstres.

Qu'ils soient grands et gris, petits et marrons, minuscules, gigantesques, il a en avait partout.

Et c'est à ce moment là qu'ils chargèrent, tous ensemble. Mais Athéna fit que leur roi, un toreau couronné et bipède, apparut, et défia Ulysse. Celui-ci accepta et le duel commença.

Le roi commença par charger Ulysse qui l'esquiva. Et lui coupa la queue. Le royal taureau chargea Ulysse une seconde fois. Mais celui-ci la décapita. Alors tous les monstres coururent après Ulysse, qui fuit, accompagné de ses compagnons vers son bateau. Ils prirent la mer et s'enfuirent de cette île maudite. »

Dans ces deuxièmes productions, certains syntagmes nominaux se rapprochent des épithètes homérique parce qu'ils font référence à des caractéristiques particulières des personnages présentés par les élèves, comme la fonction de roi attribuée au taureau par Chloé dans l'association «le royal taureau » ou parce qu'ils reprennent fidèlement des épithètes employées par Homère comme celle qu'utilise Dylan à propos d'Ulysse, "le hero au mille ruse ». Un syntagme reste marginal, celui employé par Antoine, « l'ile de forestudon ». Mais ce type d'emploi ne peut pas vraiment être assimilé à une épithète homérique dans la mesure où il concerne un lieu et non un personnage, héros ou dieu, ni même une ville, telle Troie. Pour ce qui est de la deuxième copie d'Antoine, on ne peut donc pas considérer qu'elle présente un emploi d'épithète homérique.

Ainsi, ces deuxièmes productions ne présentent plus d'emplois erronés eu égard au critère d'ordre référentiel. L'emploi des épithètes dans le contexte de leur propre 
écrit ne présentent pas de difficultés d'ordre sémantico-référentiel. La construction des syntagmes reproduit elle aussi celle des épithètes employées par Homère. Leur production s'en trouve valorisée. Le recours à un emploi explicitement demandé a permis des essais, qui ont pu être parfois peu pertinents ${ }^{(3)}$. Les élèves devant employer des épithètes, ils les ont utilisées abondamment et pas toujours de manière efficiente en effet. Mais, comme nous l'avons évoqué plus haut, dans la mesure où ces essais sont commentés par l'enseignante, voire les pairs, ils ouvrent la voie vers une appropriation plus assurée pour un réemploi pertinent ${ }^{(4)}$

Par ailleurs, dans cette deuxième production certains élèves ont très bien su associer certaines épithètes employées par Homère à des personnages différents. Ainsi « subtil » qui est relatif à Pénélope dans le texte d'Homère a pu être attribué à Ulysse dans une production où le héros ruse pour sortir d'un mauvais pas. Dans ce cas, le transfert de qualification effectué par les élèves leur permet de proposer des emplois pertinents des épithètes ainsi produites.

A l'issue de l'analyse de ces différentes productions écrites, nous remarquons donc que les élèves ont eu des difficultés concernant la construction de la référence dans le contexte de l'œuvre, problème lié à des difficultés de compréhension relatives à l'étude de ce texte d'Homère et qui n'a pas toujours été résolu. En revanche, les deuxièmes productions montrent que le transfert de qualification et le travail sur la construction du syntagme ont pu être productifs d'épithètes. Les élèves ont alors effectué des tentatives plutôt bien venues, même si elles sont encore timides. Mais, afin que l'appropriation soit plus assurée, il s'agit aussi, au cours de ce dispositif, de parler, dire, réfléchir ensemble aux réussites ainsi qu'aux difficultés et aux moyens de les résoudre.

\subsubsection{Les interactions verbales comme facteur déterminant de l'accès lexical en situation de production écrite}

L'enseignante des classes avec lesquelles nous avons travaillé favorise et suscite les interactions verbales de manière constante. Si nous retranscrivons certains échanges de classe et que nous les comparons avec quelques copies d'élèves, nous nous rendons compte de l'impact de ce recours au langage, aux interactions verbales, dans les apprentissages. Ainsi en est-il pour l'adjectif « infortuné », peu courant pour les élèves, comme nous le montre cet extrait 2 :

\section{Extrait 2 :}

P J'aimerais qu'on s'interroge sur les raisons pour lesquelles Homère a choisi de telles désignations. Parfois c'est difficile, mais parfois c'est simple et évident.

E Zeus le tout puissant qui tonne dans le ciel parce que c'est un dieu, le dieu du tonnerre.

P Oui, donc là tout à fait, ça fait référence à sa fonction, la fonction de Zeus qui tonne dans le ciel.

E Athéna c'est la fille de Zeus c'est pour ça qu'il l'appelle...

$P$ Oui qu'il l'appelle comment? Quelle dénomination tu as choisie?

$E \quad$ (cherche dans le texte)... Heu... l'illustre déesse.

$P \quad$ Ah, c'est parce que c'est la fille de Zeus qu'il l'appelle illustre déesse?

(3) D'autres exemples peuvent être donnés tels «le voyageur Ulysse» pour des raisons liées à la construction syntagmatique, ou «Ulysse le tout puissant qui ébranle la terre » pour des raisons d'ordre référentiel.

(4) Par exemple « le Soleil aux doigts rose », ou bien « le subtil Ulysse ». 
E Non, "Athéna fille de Zeus».

$P$ Oui, là c'est par rapport à son lien avec le dieu des dieux.

E L'infortuné Ulysse c'est parce qu'il a pas d'argent.

$P \quad$ Ah, alors ici est-ce que ça a un rapport avec l'argent? C'est vrai fortune / fortuné sont de la même famille. Mais ici, la fortune?

E La chance

$P$ Oui, alors infortuné dans le texte?

E C'est qu'il a pas de chance, des épreuves à surmonter.

Puis quand les élèves tentent de procéder à une catégorisation des épithètes homériques (celles qui sont relatives aux actions, ou aux qualités et aux défauts...) :

$$
\begin{aligned}
& \text { L'enseignante écrit au tableau } \\
& \text { - Actions } \\
& \text { - Qualités / défauts / état } \\
& \text { - Fonction / emblème(s) } \\
& \text { - Origine }
\end{aligned}
$$

Les élèves donnent des précisions concernant les qualités :

E Ulysse le généreux.

$P$ Oui.

E L'infortuné Ulysse.

$P \quad$ Ah, alors, on l'a vu... Qu'est-ce que cela veut dire?

E Qu'il est malchanceux.

Enfin lors de la correction de l'exercice qui consistait à définir, associer puis employer dans une phrase :

$P$ Oui, pour infortuné?

Que veut dire infortuné?

E Qui n'a pas de chance.

$P$ Oui. Une phrase?

E L'infortuné Cyclope.

$P$ Oui, alors fait une phrase qui nous montre pourquoi il peut être infortuné.

$E \quad$ L'infortuné Cyclope fut aveuglé par Ulysse.

P Oui...

E L'infortunée Pénélope attend Ulysse.

$P \quad O u i$, mais pourquoi est-elle vraiment infortunée?...

E L'infortunée Pénélope attend Ulysse depuis dix ans.

$P \quad$ Depuis?...

E Vingt ans.

P Oui, depuis vingt ans! L'infortunée Pénélope attend Ulysse depuis vingt ans!

Les élèves donnent d'autres exemples.

L'enseignante revient donc trois fois sur le problème lié à « infortuné »; elle cherche à chaque fois à revenir sur la définition de cette unité lexicale. D'abord en faisant référence à sa " famille » comme cela est rappelé, ensuite par retour sur ce qui a été dit afin de s'assurer de la maitrise du sens d'« infortuné » dans le texte d'Homère. Enfin, le recours au contexte et à l'histoire pour justifier les emplois des épithètes homériques est constant et il s'effectue via les interactions entre les élèves, leurs recherches dans la mémoire qu'ils ont du récit. Dès la deuxième partie de l'extrait les élèves reprennent le sens relevé dans la première séance. Puis, à la fin de cet extrait 2 , l'enseignante les invite à proposer eux-mêmes des phrases qui permettent de compren- 
dre l'emploi de l'épithète. Les emplois qui sont proposés pour « infortuné » deviennent plus cohérents par rapport au récit de ce chapitre 1, bien que subsiste une incohérence relative à la position énonciative. Si c'est Ulysse qui raconte dans le texte d'Homère, il n'est donc pas tout à fait exact de ce point de vue-là de proposer que le Cyclope soit « infortuné ». Ainsi par trois fois, l'enseignante revient sur cet adjectif peu courant pour les élèves et, en favorisant leur réflexion commune, elle permet une appropriation progressive de cette unité lexicale et de son sens dans le contexte de l'œuvre.

Une difficulté à propos d'« infortuné » survient également dans l'autre classe, au moment où les élèves effectuent le relevé des groupes désignant les personnages présents dans leur extrait de texte, l'enseignante insiste déjà sur cet emploi dans cet extrait 3 :

\section{Extrait 3 :}

$P$ Alors, le groupe de France.

E La déesse, Ulysse, Poséidon qui ébranle la terre, Ulysse, Calypso, Ulysse, le puissant qui ébranle la terre, Athéna, Zeus, Ulysse, Ulysse, Ulysse.

$P$ Oui. A un moment, quand tu as dit Ulysse, c'est plutôt au début...

E ... l'infortuné Ulysse.

$P$ Ah! C'est l'infortuné Ulysse à un moment!

E Il y a aussi Athéna fille de Zeus.

P Oui, Athéna fille de Zeus.

Dans cet extrait, l'enseignante ne fait que susciter l'emploi de l'adjectif que l'élève retrouve aisément dans son texte. Mais dans cette même classe, l'emploi d'« infortuné » revient une nouvelle fois durant une séance suivante lors de l'exercice de définition, association et emploi. L'extrait 4 reproduit l'échange qui s'en suit :

\section{Extrait 4 :}

$P \quad$ Infortuné?

E L'infortuné Cyclope mange les compagnons d'Ulysse.

$P \quad$ L'infortuné Cyclope mange les compagnons d'Ulysse. Alors, est-ce que dans cette phrase on voit qui est infortuné?

Qu'est-ce que ça veut dire infortuné? Essaie de te rappeler, on l'a défini.

E Qu'il na pas de chance.

$P$ Oui, alors dans cette phrase est-ce qu'on sait pourquoi il n'a pas de chance? $C^{\prime}$ 'st vrai qu'il est infortuné, comment on pourrait le dire alors?

E L'infortuné Cyclope s'est fait prendre par la ruse d'Ulysse parce que Ulysse lui fait une ruse.

$P \quad$ Oui, laquelle pour être plus précis?

E Il lui plante le pieu dans l'oil.

$P$ Oui, alors on aurait plutôt l'infortuné Cyclope s'est fait crever l'œil par Ulysse.

E L'infortunée Pénélope.

$P$ Oui, sur la construction pas de problème. Infortunée, terminaison?

E -ée.

P Oui parce que c'est Pénélope.

E L'infortuné Pénélope car elle attend Ulysse pendant dix ans.

$P$ Oui, est-ce que tu as besoin de dire CAR elle attend Ulysse pendant dix ans?

E Non.

$P \quad$ Alors vas-y construit correctement ta phrase. L'infortunée Pénélope...

E L'infortunée Pénélope attend Ulysse pendant dix ans. 


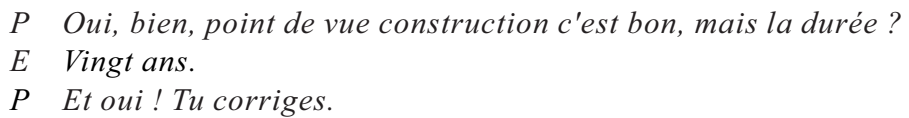

Dans cet échange, l'enseignante précise bien qu'elle revient sur une définition qui a été donnée. Une sorte de répétition s'opère, comme dans l'autre classe et les élèves retrouvent la même unité, mais la travaillent différemment. Les interactions suscitées par l'enseignante incitent les élèves à recourir eux-mêmes au sens du texte. Lors de ces interactions ils sont toujours amenés à employer l'épithète dans une phrase qui pourrait en justifier le sens, et ce par rapport au récit. Ce recours constant au contexte apparaît essentiellement lors des interactions, quand l'enseignante demande aux élèves de justifier leur emploi en utilisant les événements du récit. Pour autant, la même difficulté survient quant à la pertinence de l'utilisation d'« infortuné » à propos du Cyclope. Néanmoins, il semble que ce travail d'association mené en parallèle à une réflexion sur le contexte d'emploi dans l'œuvre, permette une appropriation du lexique. En effet, cette unité lexicale, peu connue des élèves, est employée à plusieurs reprises dans leur dernière production, alors qu'elle n'apparaissait pas dans les reformulations initiales. Par exemple dans le texte de David :

«L'infortuné Ulysse etant sur son bateau avec ses 6 fidèles compagnons. Il aperçu au loin une île habité, il décida donc de s'y rendre, il arriva sur l'île. Celle-ci était entouré de rempart. Il all frapper, mais personne n'ouvrit, alors il décida de l'enfoncer avec un bélier, il prit un bélier avec ses compagnons et enfoncèrent la porte.

Il rentra avec ses courageux compagnons. Et vit que la ville était vide. Puis il entendit un grognement.

Il se retourna et vit un monstre, il dégaina alors son épée et lui trancha la gorge, la tête tomba puis le corp. Tout à coup, il entendi un signal d'alarme et des monstre sortirent de partout, ils se précipitèrent sur lui. Il dit a se compagnons « venez m'aider à les touer passer devant, je vous couvre avec mon arc ", mais le plus fidèle et infortuné compagnon se fit coupé la tête par un monstre, alors Ulysse s'énerva et Ulysse, Athéna et ses compagnons restants tuèrent le reste des monstres ».

« Infortuné » est également employé par Clémence :

« Pendant le voyage d'Ulysse, lui et se six compagnons accostent sur une île à l'air paradisiaque. Une île qui paraissait plutôt banale à vue d'œil.

Ulysse qui ne craint rien, quitte le bateau avec quelques un de ses compagnons, les autres restent près du bateau. Ils avancent dans la forêt tropicale. Ils entendent des bruits et se cachent. Là, ils découvrentune gigantesque créature ideuse et repoussante. Elle a trois jambes très poilus avec, au bout, en guise d'orteils, de géantes griffes pointues, tout son corps est remplis d'épines et a de très grandes ailes de chauve-souris. Sa tête, elle, au contraire, est toute petite avec quatres énormes yeux. Sa bouche laisse dépasser deux longues dents et son petit nez de cochon fait un bruit insuportable. Sa peau est toute verte et couverte de grosses verrues. On peut voir de tous petits bras sous ses ailes. L'infortuné Ulysse est remarqué par cette affreuse bête. Elle prend Ulysse par le pied, l'examine bien et le cogne contre un rocher pour l'assomer et fait de même avec les compagnons.

A leur réveil, les malheureux d'Ithaque se retrouvent dans une cage pendue au sommet de ce qui semblait être un tipi en feuille d'arbre. Le monstre qui a trois jambes se tient debous, en cuisinant dans une marmite. Il s'approche de la cage et 
prend un des compagnons d'Ulysse en ricanant, puis le plonge dans la géante marmite. Attendant que le tout cuise, il va faire un tour dehors. Un petit enfant monstre surgit dans celle-ci. Ulysse le rusé remarque une géante sucette posé sur un meuble. Il le dit a se compagnons qui lui font une courte échelle pour l'attraper et ils font ensemble un marché avec l'enfant : les habitants d'Ithaque lui donnent la sucette contre la clef de la cage. L'enfant accepte et Ulysse part.

Ulysse et ses cinq compagnons reprennent la route et s'en alèrent. »

Dans ces deux productions, l'épithète « infortuné » est employée de manière cohérente par rapport au récit qui est proposé par les élèves. Ainsi dans la production de David, Ulysse est effectivement infortuné de voguer indéfiniment et son "fidèle " compagnon " infortuné » de s'être fait couper la tête, même si ici la construction du groupe « le plus fidèle et infortuné compagnon » est à reprendre. De même dans la production de Clémence, Ulysse est en effet malchanceux d'avoir été remarqué par le monstre. Dans cette dernière production, nous pouvons même remarquer que le travail mené sur le lexique semble inciter les élèves à recourir à la substitution, ainsi "les malheureux d'Ithaque " désignant Ulysse et ses compagnons. Cette réflexion sur le sens et l'emploi d'« infortuné » s'est bien effectuée en classe via les interactions verbales, comme nous le montrent les transcriptions, et ce moment de réflexion a ouvert la voie à l'emploi en situation de production. Il semble donc bien qu'en l'occurrence le travail mené oralement favorise l'accès lexical en situation de production écrite.

Les deux productions ci-dessus montrent aussi que les interactions permettent aux élèves de recourir à la notion de ruse concernant Ulysse. Nous reproduisons en annexe 3 d'autres productions se référant à cette notion de ruse, dans lesquelles les élèves se risquent même à utiliser "Ulysse aux mille ruses". Le travail effectué à propos des épithètes homériques, comme par exemple « aux mille ruses », permet également aux élèves de se saisir d'un aspect du caractère du personnage qu'ils vont réutiliser dans leur production, par exemple en apportant des précisions telle «comme il était rusé...». Le lien étroit entre l'enseignement-apprentissage du lexique et la compréhension, que nous avons déjà mentionné, réapparait ici.

Par ailleurs, un autre point est à relever concernant ces interactions verbales. L'enseignante a insisté tout au long du dispositif sur les différents aspects du lexique, définitionnel, étymologique, syntagmatique, contextuel. Ainsi lorsque les élèves proposent des groupes nominaux, la question est toujours de voir « si c'est bon du point de vue de la construction et du point de vue du sens ». Dans l'extrait d'échange ci-dessus (extrait 4), nous remarquons d'ailleurs qu'elle précise « oui, sur la construction, pas de problème ». Mais dans l'autre classe, cette construction a été mise à mal dans le travail oral au moment où un élève propose une épithète pour l'un de ses camarades, ainsi dans cet extrait 5 :

Extrait 5 :

E Théo le motard.

P Bon, si c'est vrai, ça va pour la construction...

E Christopher et ses chaussettes.

$P \quad$ Est-ce que ça correspond à une épithète homérique?

Les élèves regardent au tableau les différentes constructions possibles.

$P \quad$ Vous vous rappelez on avait dit pour Céline...

E Ça n'allait pas, Céline à... et pas Céline et...

$P$ Oui, on avait dit que la construction allait mieux si on disait Céline à la belle che- 
velure. C'est un problème de construction ici.

Donc Mourad...

E Ben Christopher avec ses chaussettes...

E Non à!

E ...A ses chaussettes

E (tous) Aux!

E Aux chaussettes

$P$ Oui, aux chaussettes, mais si on dit Christopher aux chaussettes, est-ce que ça semble pertinent?

E (Mourad) Noires!

$P$ Voilà, il vaut mieux rajouter une précision.

E (Mourad) Christopher aux chaussettes noires.

E Mais dans le messager rapide et clair, il y a bien " et" » aussi.

$P$ Oui, mais dans ce cas le « et » sert à quoi Virginie? Il sert à relier les deux caractéristiques (l'enseignante reprend les GN écrits au tableau pour expliquer cela). Où il ne faut pas de " et " c'est après l'identité du personnage. Par exemple là on a "la nymphe AUX cheveux bouclés", mais on ne dit pas la nymphe ET ses cheveux bouclés, ça ne correspond pas à une construction d'épithètes homérique. Le "et " sert à relier les deux adjectifs. On ne dit pas Hermès et sa baguette d'or, mais "Hermès à la baguette d'or ".

E Audrey et sa fourrure

Heu, non, Audrey à la fourrure

$P \quad$ Oui, par rapport à son pull...

E Victor aux grandes dents

Nous retrouvons dans cet échange les difficultés liées aux associations entre les unités lexicales composant les groupes nominaux afin d'obtenir des épithètes homériques, ce type d'association étant davantage propre au langage écrit dans la traduction d'Homère qu'au langage oral courant pour les élèves. Néanmoins, nous avons vu lors de l'analyse des deuxièmes productions écrites que le travail sur les épithètes aura permis aux élèves d'oser des associations lexicales propres à caractériser leurs personnages. Mais dans l'échange ci-dessus, c'est par l'étayage que l'enseignante parvient à faire évoluer la construction proposée par Mourad qui de "Christopher aux chaussettes » devient finalement "Christopher aux chaussettes noires ». Par ailleurs, cet exercice oral permet aux élèves de soulever des questions concernant cette construction que l'enseignante explique alors. Les dernières répliques montrent que les élèves se saisissent petit à petit de ce travail sur la construction, à travers l'hésitation de l'élève qui corrige elle-même spontanément sa proposition. Il s'avère ainsi que cet exercice uniquement oral effectué dans cette classe permet essentiellement de revenir sur la construction grammaticale du groupe nominal. Néanmoins, même dans l'autre classe n'ayant pas pratiqué cet exercice oral mettant l'accent sur les constructions, les dernières productions présentent fort peu de constructions erronées. En effet, dans la classe A qui n'a pas pratiqué l'exercice oral, dix-sept élèves sur vingt-et-un ont eu recours à des épithètes homériques, alors que dans la classe $\mathrm{B}$ qui a pratiqué l'exercice oral, douze élèves sur vingt ont recouru à des épithètes homériques dans leur écrit.

Le travail réalisé à l'écrit a sans doute favorisé les apprentissages, d'autant que les interactions lors des corrections étaient toujours abondantes comme nous l'avons déjà remarqué. Si l'analyse des interactions et leurs comparaisons avec les copies des élèves nous ont permis de montrer le lien entre interactions verbales et accès lexical 
en production écrite, comme pour le cas problématique de l'adjectif «infortuné »employé par les élèves dans leur deuxième production, la comparaison entre les deux classes semble montrer qu'un travail écrit reste favorable aux apprentissages, compte tenu du fait qu'il est soutenu par les interactions verbales.

Il semble donc que dans ces deux classes, ce soit davantage l'influence réciproque des interactions verbales et des exercices écrits qui a favorisé l'accès lexical en situation de production écrite, plus que l'exercice oral. En effet, si l'on considère que l'appropriation s'effectue d'abord en compréhension, à l'oral, puis à l'écrit, chaque classe a respecté cette chronologie. Lors des reformulations et de la remise en ordre du texte, la compréhension a été exercée ; lors des interactions verbales durant les séances, et lors des différentes productions écrites qui ont jalonné le dispositif, chaque étape de l'appropriation apparait. La comparaison entre les épithètes travaillées oralement en classe via les interactions et celles employées par les élèves dans les productions 1 et 2 montre d'ailleurs le lien étroit entretenu par ces deux aspects du dispositif. Nous retrouvons en effet dans les productions les épithètes abordées oralement lors des interactions verbales. Les élèves ont ainsi employé des constructions syntagmatiques correspondant à celles des épithètes homériques et ils ont repris certaines formules du texte comme « l'infortuné Ulysse » sans incohérence sémantico-référentielle. Ce constat nous permet d'avancer qu'en effet la production écrite est un élément déterminant de l'appropriation lexicale et que les interactions verbales, liées à des exercices écrits, semblent favoriser l'accès lexical en situation de production écrite.

\section{Références}

CAlaque, E. (2000) : «Enseignement et apprentissage du vocabulaire». Calaque, E. \& Grossmann, F. (coord.), Enseignement / apprentissage du lexique. LIDIL $n^{\circ} 21$, pp. 17-36. Université Stendhal Grenoble 3. Grenoble.

ChANFRAUlT-DucheT, M.-F. (2004) : «Vers une approche syntagmatique du lexique en didactique du français ». Calaque, E. \& David, J. (coord.), Didactique du lexique : contextes, démarches, supports, pp. 103-114. De Boeck éditions. Bruxelles.

DAVID, J. (2000) : « Le lexique et son acquisition : aspects cognitifs et linguistiques. » Le français aujourd'hui 131, pp. 31 à 40. Association Française des Enseignants de Français. Paris.

DAVID, J., DUBOIS, D., FAYOL, M., RÉMOND, M.(coord.). (2000) : Maîtriser la lecture : poursuivre l'apprentissage de la lecture de 8 à 11 ans. CNDP. Éditions Odile Jacob. Paris.

Fillmore, C. J. (1968) : «The Case for Case » (1968) : Bach \& Harms (coord.), Universals in Linguistic Theory, pp. 1-88. Holt, Rinehart, and Winston. New York.

Grossmann, F. \& BOCH, F. (2004) : "Production de textes et apprentissage lexical : l'exemple du lexique de l'émotion. ". L'observation réfléchie de la langue à l'école, Repères $n^{\circ} 28$, pp. 117-135. INRP. Lyon.

GuIMIER, C. (coord.) (1996) : Co-texte et calcul du sens. Presses universitaires de Caen. Caen.

LEGRAND, G. (2004) : «Les rubriques du « vocabulaire » dans l'enseignement élémentaire : étude sur deux siècles de textes ministériels ». Calaque, E. \& David, J. (coord.), Didactique du lexique : contexte, démarches, supports, pp. 19-34. De Boeck éditions. Bruxelles. 
MAINGUENEAU, D. (1996, 2009) : Les termes clés de l'analyse du discours. Éditions du Seuil. Paris.

MEL'ČUK, I. (2004) : "Actants in Semantics and Syntax ». Linguistics, 4. http ://olst.ling.umontreal.ca (dernière consultation le 17.07.2012).

Ministère De L'ÉDUCATION NATIONALE. Programmes du cycle des approfondissements. Bulletin Officiel hors-série $\mathrm{n}^{\circ} 3$ du 19 juin 2008. http ://eduscol.education.fr/pid23391/programmes-ecolecollege.html_(dernière consultation 14.03.2011).

- Programmes du collège. Bulletin Officiel spécial $n^{\circ} 6$ du 28 août 2008. http://eduscol.education.fr/pid23391/programmes-ecole-college.html (dernière consultation 14032011$)$.

PAVEAU, M.-A. (2000) : "La "richesse lexicale" entre apprentissage et acculturation ». Le français aujourd'hui 131, pp. 19-30. Association Française des Enseignants de Français. Paris.

PETIT, G. (2000) : «Didactique du lexique : état d'une confusion ». Le français aujourd'hui 131, pp. 53-62. Association Française des Enseignants de Français. Paris.

Picoche, J. (1986) : Structures sémantiques du lexique français. Nathan. Paris. - (1993) : Didactique du vocabulaire français. Nathan. Paris.

POLGUÈRE, A. (2004) : Les fonctions lexicales pour tous. OLST, département de linguistique et de traduction Université de Montréal. http ://w3.u-grenoble3.fr(dernière consultation 14 mars 2011).

- (2008) : Lexicologie et sémantique lexicale : Notions fondamentales. Les Presses de l'Université de Montréal, collection Paramètres. Montréal.

SARDiER, A. \& Grossmann, F (2010) : «Comment favoriser le réemploi lexical ?». Lexique Vocabulaire, Recherches $n^{\circ} 53$ pp. 9 à 29. Lille.

TESNIÈRE, L. (1959) : Éléments de syntaxe structurale. Klincksieck. Paris.

\section{ANNEXES}

\section{ANNEXE 1}

\section{Exemples d'exercices d'association proposés aux élèves}

\section{— Voici des épithètes homériques : en associant chacune d'elles au nom qu'elle} enrichit, construis un groupe nominal.

à la baguette d'or - amère - aux belles tresses - aux doigts roses - ailées - divin haute - profonde - la plus sage des femmes - aux cheveux bleus.

Ulysse - Pénélope - Poséidon - Hermès - La déesse - La flèche - une caverne Une montagne - Des paroles - Aurore.

a) Cherche dans un dictionnaire le sens des adjectifs qualificatifs de la liste A que tu ne connais pas.

b) Associe un ou plusieurs de ces adjectifs à chacun des personnages homériques de la liste $B$.

c) Emploie dans une phrase les groupes nominaux ainsi formés

A : arrogant - cruel - généreux - infortuné - prudent - sage - subtil - terrible vénérable.

B : Alkinoos - Antinoos - Calypso - Le Cyclope - Pénélope - Télémaque. 


\section{ANNEXE 2}

\section{Consigne d'écriture demandant explicitement l'emploi}

d'épithètes homériques

\section{Travail d'écriture \\ Les épithètes homériques}

Sujet : Athéna protège Ulysse et l'aide à rejoindre Ithaque. Lors du séjour d'Ulysse sur l'île des Phéaciens, Aphrodite intervient et voudrait qu'il tombe amoureux de Nausicaa qui n'est pas insensible à son charme.

Imagine un nouvel épisode de l'Odyssée mettant en scène ces quatre personnages.

Consignes d'écriture :

Emploie pour chacun des personnages des épithètes homériques variées.

\begin{tabular}{|l|l|l|}
\hline Cohérence du récit & $/ 5$ & \\
\hline Epithètes homériques & $/ 8$ & \\
\hline Expression & $/ 7$ & \\
\hline Note $/ 20$ & $/ 20$ & \\
\hline
\end{tabular}

\section{ANNEXE 3}

Consigne d'écriture et exemples de productions d'élèves se référant à la notion de ruse à l'issue du dispositif (nous soulignons)

\section{Travail d'écriture Un nouvel épisode de l'Odyssée}

Sujet Ulysse, avec les six compagnons qui restent, accoste une nouvelle île. Il découvre ses habitants qui sont des monstres. Ulysse devra mettre en œuvre toutes ses qualités pour les affronter. Racontez.

\section{Plan à suivre}

- Arrivée sur l'île

- Découverte des habitants

- Affrontement

- Départ de l'île

N.B. N'oublie pas que les dieux sont omniprésents dans l'Odyssée.

Nathan

«Ulysse et se compagnons arrive sur une très grosse île nommer Usutios, l'île mystérieuse.

Ils furent très mal accueillit par des monstres « aigre » mi-oiseau mi tigre.

Les habitants de cette île les transformèrent en esclaves mais le rusé Ulysse su que les aigres ne voyait pas la nuit.

Ils décidèrent donc de partirent la nuit mais les aigres flérait très bien. Les aigres pourchassèrent Ulysse et ses compagnons mais Ulysse réussirent à couper le nez de chacun des aigres, du coup ils s'entretuèrent et Ulysse et ses compagnons partirent. »

\section{Nouaman}

«Ulysse était bientôt arrivé sur l'île. La ravissante Athéna par à Ulysse et lui dit Ulysse fait attention quant tu arrivera sur cette île. Elle s'appelle l'île des gumgum. 
Ulysse le rusé arrivé sur l'île.

Ulysse et ces compagnons en vue un horrible monstre, il était affreux : il avait une grande tête, quatre yeux, deux bouche, trois nez, un pied, une main qui porté de ma poitrine, et il était énorme, c'était affreux. Le monstre qu'a vue Ulysse était paser devant eux, le monstre regarda Ulysse avec une aine arreyante il avança et prie deux compagnons d'Ulysse et les manga en même tant et en reprie trois et repartir en courant il manga en compagnont pendant la route il a la prévenir son maître et parla au compagnon d'Ulysse et leur dit d'allez prévenir l'autre chez et dit-lui quand va tous vous tué pour être venue su cette île sacré. Il relachèrent les deux compagnons d'Ulysse pour aller prévenir est il dit à Ulysse qu'il allait tous nous tué ce sont des monstre. De nouveau la déesse de la beauté Athéna rapparu et dit a Ulysse qu'elle lui donnera une épée magique qui peut tuer tranche d'un seul coup, tu pourra tué les monstre avec l'épée. Les monste arrive le chez alla voir Ulysse et prie les trois compagnons et le manga. Ulysse prie l'épée et les en tous tranche.

Le lendemain matin Ulysse à la prendre des provision chez le monstre et reparti de l'île gumgum. »

Elina

« L'île mystérieuse.

Ulysse et ses compagnons montèrent sur le bâteau, Ulysse le conduit pendant que se compagnons mettent les voiles, lèvent l'ancre. Athéna surveilla pour leur venir en aide si un problème se posait. Deux jours et deux nuits, quand ils arrivèrent sur une île mistérieuse : ils n'entendirent pas un bruit et ne virent personne. Ils mettait l'ancre, enlever les voiles et sortirent du bâteau.

Ils alèrent chercher du bois quand soudain, ils appercurent des ombres, alors ils approchèrent et plus ils avancèrent plus ils entendtient du bruits. Soudain, ils virent une cage alors ils avancèrent, puis ils virent un groupe d'homme enfermé. Alors Ulysse eut pitié et les libéra. Ulysse leurs demandait :

«-Qui êtes-vous? Qui vous a enfermé ici ? Pourquoi êtes-vous là ?

- Nous sommes les Arborigènes et des monstres nous a enfermé dans cette cage mais nous sommes juste venus ici car nous voulions juste faire un pause » répondit le chef de clan.

Ils continuèrent leur voyage ensemble. Quelques mètres plus loin, ils virent plein de monstres. Les Arborigènes les reconnurent. Ulysse aux milles ruses expliqua son plan :

«- Ils son grand, gros et pas très intelligeants donc nous n'avons qu'à les bloqués dans le petit chalet et partir. »

Ulysse et se compagnons couraient et les monstres les poursuivirent. Ulysse fait entré ses amies et les monstres. Et les monstres furent bloqués ne pouvant ni sortir, ni rentrer.

Mais Ulysse et se compagnons avec les Arborigènes étaient aussi enfermés dans le chalet mais Ulysse eut l'idée de creuser et Athéna leurs donna la force et le courage de creuser.

Ulysse et se compagnons repartirent sur le bâteau et les Arborigènes remercirent Ulysse en lui offrant, en guise de souvenir, un attrappe cauchemar qu'il fallait accrocher sur son lit et ils ne feraient plus que bons rêves. » 